\title{
Agonist Versus Antagonist in Intracytoplasmic Sperm Injection Cycles: Which Is the Best?
}

\author{
Bushra J. Al-Mousawi ${ }^{1}$, Maanee A.H. Al-Azzam ${ }^{1}$, Balsam Al-Zahawi ${ }^{1}$, Hayder A. Fawzi ${ }^{*}$
}

\begin{abstract}
Objectives: The comparison of gonadotropin-releasing hormone $(\mathrm{GnRH})$ agonist against $\mathrm{GnRH}$ antagonists regarding the pregnancy rate and effect of various predictors on pregnancy outcomes.

Materials and Methods: This prospective comparative study involved 189 women who underwent intracytoplasmic sperm injection (ICSI) cycles and were divided into agonist (107 patients) and antagonist arm (82 patients) groups. The chemical and clinical pregnancy rate was the main outcome and the other outcomes included the number and quality of oocyte measurement and pregnancy outcomes.

Results: Based on the results, the agonist protocol showed a higher rate of pregnancy (32.7\%, 95\% CI: $23.9-42.4 \%)$ compared to the antagonist protocol $(22.0 \%, 95 \%$ confidence interval $(\mathrm{CI}): 13.6-32.5 \%)$ with the odds ratio $(\mathrm{OR})$ of $(95 \% \mathrm{CI})=1.73(0.89-3.35)$. The results further revealed that the count of retrieved oocytes, count of M2 oocytes, count of fertilized oocytes, count of embryos, and the fertilization percent out of total retrieved oocytes were higher in the agonist arm compared to the antagonist arm. In the multivariate analysis after adjusting for the confounders, the agonist protocol had higher odds of a successful pregnancy compared to the antagonist protocol by $57 \%$ (partial OR $=1.57, P$ value $=0.23$ ).

Conclusions: In general, the agonist protocol offers a favourable outcome in comparison to the antagonist arm, and there seems to be an intrinsic benefit for the agonist protocol, which is not explained by the higher number of transferred embryos.

Keywords: GnRH agonist, GnRH antagonist, Pregnancy rate, In vitro fertilization, ICSI
\end{abstract}

\section{Introduction}

Infertility is considered as a devastating issue that can cause suffering to any couple, with a prevalence of $15 \%$ in the first year of marriage (1). The gonadotropin-releasing hormone ( $\mathrm{GnRH}$ agonist) has been a major asset in the assisted reproductive technology since its development in the 1980s $(2,3)$. In more than 2 decades, the $\mathrm{GnRH}$ agonist became the "gold standard" in ovarian stimulation and thus was used in the long protocol with the purpose of an initial increase in the production of luteinizing hormone ( $\mathrm{LH})$ and follicle-stimulating hormone (FSH) from the pituitary gland. Accordingly, these changes cause a reduction in the stimulation of ovary, the suppression of folliculogenesis, and a decline in circulatory estrogen to the menopausal level within three weeks by maintaining the continuous administration of the GnRH agonist leading to the down-regulation of $\mathrm{GnRH}$ receptors and consequently the lowering of $\mathrm{LH}$ and FSH (4-7). The prevention of the early $\mathrm{LH}$ surge leads to a reduction in the risk of ovarian hyperstimulation syndrome (8), which is related to the ability of the $\mathrm{GnRH}$ agonist to desensitize the pituitary gland (9).

According to some studies (10-12), the GnRH antagonist was first used in the late 1990s aiming at reaching more friendly in vitro fertilization (IVF). In addition, the GnRH antagonsit offered several advantages such as reducing the total number of the applied gonadotropins, the risk of ovarian hyperstimulation syndrome, and the costs, as well as having low side effects and shorter treatment durations (6).

The binding of GnRH agonist to a receptor on pituitary causes the desensitization of pituitary and consequently the down-regulation of gonadotropin secretion $(13,14)$. Further, the GnRH agonist initially has stimulatory effects, followed by subsequent reductions in gonadotropin levels (14). On the other hand, the GnRH antagonist produces the immediate inhibition of gonadotropin secretions thus it can be given after the initiation of gonadotropins in IVF protocols (15).

This study compared two different protocols of controlled ovarian hyperstimulation $(\mathrm{COH})$ in order to determine which protocol leads to better outcomes in terms of pregnancy rate and identify the predictors of the higher rate of pregnancy. Finally, the study sought to examine the quality of oocytes (i.e., number, fertilization, and the like), as well as the embryo retrieval rate and quality between these two protocols.

Received 10 May 2019, Accepted 14 September 2019, Available online 1 October 2019

${ }^{1}$ Reproductive Medical Center of Fertility and in vitro fertilization, Kamal AL-Samaria Hospital, Baghdad, Iraq. ${ }^{2}$ Department of Pharmacy, Al-Rasheed University College, Ministry of Higher Education and Scientific Research, Baghdad, Iraq.

*Corresponding Author: Hayder A. Fawzi, Tel: +964 (0)7722627943, Email: hayder.adnan2010@gmail.com 
Key messages

- The agonist protocol showed higher pregnancy rates compared to antagonist protocol.

- The agonist protocol showed higher Embryonic outcomes.

- The agonist protocol showed higher successful pregnancy rates compared to antagonist protocol.

\section{Materials and Methods}

Study Design

This prospective comparative study including 226 IVF cycles was conducted at the Reproductive Medical Center of Fertility and IVF cycle in Kamal AL-Samaria Hospital (Baghdad/Iraq) from November 2014 to November 2015. In this study, 122 women were given the agonist protocol while 104 others received the antagonist protocol. Of those women, 15 and 22 cases discontinued the program from each group, respectively (Figure 1).

\section{Inclusion Criteria}

Women within the age range of 18-45 years undergoing their $1^{\text {st }}$ or $2^{\text {nd }}$ cycle of IVF regardless of their indication for infertility were included in this study.

\section{Sample Size Calculation}

The sample size was computed 91 women in the two arm groups based on a previous study by Orvieto et al (16) in which they reported $41.2 \%$ and $25.3 \%$ pregnancy rates in agonist and antagonist arms, respectively, as well as the types I and II error of $10 \%$ and $20 \%$.

\section{GnRH Agonist Protocol of $\mathrm{COH}$}

Decapeptyl 0.1 mg (Ferring, Kiel Germany), given subcutaneous on day 21 of the previous cycle, was given daily for 14 days.

\section{GnRH Antagonist Protocol of $\mathrm{COH}$}

FSH (Gonal F Merck Serono or Puregon MSD) was given on the $2^{\text {nd }}$ day with the dominant follicle size $\leq 14 \mathrm{~mm}$ and the GnRH antagonist (Cetrotide, Asta Medica AG, Frankfurt, Germany) was administered daily.

Criteria of Human Chorionic Gonadotropin Admiration Two ampules of Ovitrelle $250 \mu \mathrm{g} / 0.5 \mathrm{~mL}$ (Merck Serono S.p.A, Italy) were given in the presence of $\geq 3$ follicles $(\geq 18$

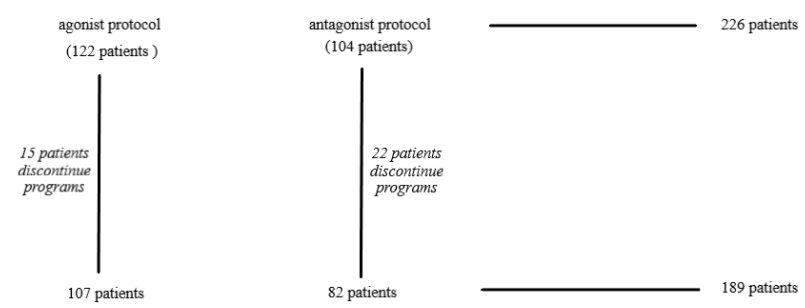

Figure 1. Study Flowchart. $\mathrm{mm}$ in diameter), and continuous elevations in the serum estradiol concentration.

Oocyte Aspiration

It was performed 34 to 36 hours after human chorionic gonadotropin injection guided by vaginal ultrasound. Moreover, intracytoplasmic sperm injection (ICSI) was performed using standard procedures as described by Rubino et al (17), with embryo transfer proceeded 2 or 3 days later. The maturation and morphological features of oocyte were investigated immediately before ICSI. Then, the fertilization rate, early embryo development, and transfer were documented and studied as well.

\section{Statistical Analysis}

All analyses were carried out using IBM SPSS 21.0 (Chicago, IL). An independent $t$ test was used to compare between two continues variables Then, partial regression analysis was used to calculate the odds ratio and its $95 \%$ confidence interval.

\section{Results}

The incidence of chemical pregnancy was higher in women who received the agonist protocol with an odds ratio of 1.73 although it did not reach statistical significance (Table 1).

The count of retrieved oocytes, count of M2 oocytes, count of fertilized oocytes, count of embryos, and the percent fertilized out of the total retrieved oocytes were significantly different (Table 2). Tables 3 and 4 present the comparison of various variables for each protocol according to the pregnancy outcome (defined as a positive or negative pregnancy test).

As shown in Table 5, using the agonist protocol increased the odds of having a successful chemical pregnancy by $57 \%$ after adjusting for confounding the effect of the M2 count, age, and transferred embryos compared to those using the antagonist protocol. Nonetheless, this estimate failed to reach a statistically significant level. There seems to be an intrinsic added benefit for the agonist protocol, which is not explained by a higher number of M2 oocytes or the higher number of transferred embryos.

Table 1. Incidence Rate of Chemical Pregnancy by the Type of the Ovulation Induction Protocol Among Subjects Who Completed the Treatment Schedule

\begin{tabular}{|c|c|c|c|c|}
\hline \multirow[t]{2}{*}{ Protocol used } & \multirow{2}{*}{$\begin{array}{c}\text { Total } \\
\mathbf{N}\end{array}$} & \multicolumn{2}{|c|}{$\begin{array}{c}\text { Chemical Pregnancy } \\
\text { (Pregnancy Test) }\end{array}$} & \multirow[t]{2}{*}{$95 \% \mathrm{Cl}$} \\
\hline & & No. & $\%$ & \\
\hline Agonist protocol & 107 & 35 & 32.7 & (23.9\% to $42.4 \%)$ \\
\hline Antagonist protocol & 82 & 18 & 22.0 & (13.6\% to $32.5 \%)$ \\
\hline Total & 189 & 53 & 28.0 & ( $21.7 \%$ to $35.0 \%$ ) \\
\hline
\end{tabular}

Note. $P$ (Chi-square) $=0.10$ [NS]; NS: Not significant; The odds ratio for chemical pregnancy in the agonist group compared to the antagonist group $=1.73(95 \% \mathrm{Cl}=0.89-3.35)$. 
Table 2. Assessment of IVF Outcomes According to Treatment Protocols

\begin{tabular}{|c|c|c|c|}
\hline \multirow[b]{2}{*}{ Outcome parameters } & \multicolumn{2}{|c|}{ Treatment Protocol } & \multirow[b]{2}{*}{$P$ value } \\
\hline & $\begin{array}{c}\text { Agonist protocol } \\
n=107\end{array}$ & $\begin{array}{c}\text { Antagonist Protocol } \\
n=82\end{array}$ & \\
\hline Count of retrieved oocytes & $10 \pm 0.5$ & $9 \pm 0.59$ & 0.019 \\
\hline Count of M2 oocytes & $7 \pm 0.46$ & $6 \pm 0.47$ & 0.02 \\
\hline Count of fertilized oocytes & $6 \pm 0.37$ & $4 \pm 0.36$ & $<0.001$ \\
\hline Count of embryos & $4 \pm 0.28$ & $3 \pm 0.33$ & 0.021 \\
\hline Count of transferred embryos & $3 \pm 0.14$ & $2 \pm 0.16$ & $0.17[\mathrm{NS}]$ \\
\hline Percent mature (M2) out of total retrieved oocytes & $68 \pm 2.42$ & $64 \pm 3.37$ & $0.25[\mathrm{NS}]$ \\
\hline Percent fertilized out of total retrieved oocytes & $58 \pm 2.71$ & $45 \pm 3.14$ & 0.001 \\
\hline Percent embryos out of total retrieved oocytes & $43 \pm 2.61$ & $39 \pm 2.99$ & $0.32[\mathrm{NS}]$ \\
\hline
\end{tabular}

Note. NS: Not significant. Data presented as mean \pm standard error.

Table 3. The Mean of Selected Outcome Parameters by Chemical Pregnancy Stratified by the Agonist Treatment Protocol

\begin{tabular}{|c|c|c|c|}
\hline \multirow{2}{*}{ Variables } & \multicolumn{2}{|c|}{ Chemical Pregnancy } & \multirow{2}{*}{$P$ Value } \\
\hline & Negative $(n=72)$ & Pregnant $(n=35)$ & \\
\hline Age (y) & $28 \pm 0.6$ & $29 \pm 0.93$ & $0.54[\mathrm{NS}]$ \\
\hline Count of retrieved oocytes & $10 \pm 0.61$ & $12 \pm 0.87$ & $0.08[\mathrm{NS}]$ \\
\hline Count of M2 oocytes & $6 \pm 0.5$ & $9 \pm 0.82$ & $<0.001$ \\
\hline Count of fertilized oocytes & $5 \pm 0.43$ & $7 \pm 0.69$ & 0.025 \\
\hline Count of embryos & $4 \pm 0.35$ & $6 \pm 0.4$ & $<0.001$ \\
\hline Count of transferred embryos & $2 \pm 0.18$ & $3 \pm 0.14$ & $<0.001$ \\
\hline Percent mature $(\mathrm{M} 2)$ out of total retrieved oocytes & $63 \pm 3.13$ & $80 \pm 2.89$ & $<0.001$ \\
\hline Percent fertilized out of total retrieved oocytes & $56+3.56$ & $63 \pm 3.83$ & $0.21[\mathrm{NS}]$ \\
\hline Percent embryos out of total retrieved oocytes & $37 \pm 3.26$ & $54 \pm 3.75$ & 0.003 \\
\hline Count of doses & $47 \pm 2.01$ & $49 \pm 1.78$ & $0.43[\mathrm{NS}]$ \\
\hline
\end{tabular}

Note. NS: Not significant. Data presented as mean \pm standard error.

Table 4. The Mean of Selected Outcome Parameters by Chemical Pregnancy Stratified by the Antagonist Treatment Protocol

\begin{tabular}{|c|c|c|c|}
\hline \multirow{2}{*}{ Variables } & \multicolumn{2}{|c|}{ Chemical Pregnancy } & \multirow{2}{*}{$P$ Value } \\
\hline & Negative $(n=64)$ & Pregnant $(n=18)$ & \\
\hline Age (years) & $31 \pm 0.81$ & $31 \pm 1.1$ & $0.85[\mathrm{NS}]$ \\
\hline Count of retrieved oocytes & $9 \pm 0.7$ & $8 \pm 1.06$ & $0.77[\mathrm{NS}]$ \\
\hline Count of M2 oocytes & $6 \pm 0.57$ & $6 \pm 0.76$ & $0.53[\mathrm{NS}]$ \\
\hline Count of fertilized oocytes & $4 \pm 0.44$ & $5 \pm 0.51$ & $0.26[\mathrm{NS}]$ \\
\hline Count of embryos & $3 \pm 0.41$ & $4 \pm 0.33$ & $0.53[\mathrm{NS}]$ \\
\hline Count of transferred embryos & $2 \pm 0.18$ & $3 \pm 0.21$ & 0.005 \\
\hline Percent mature (M2) out of total retrieved oocytes & $59 \pm 3.99$ & $79 \pm 4.36$ & 0.014 \\
\hline Percent fertilized out of total retrieved oocytes & $41 \pm 3.7$ & $59 \pm 4.23$ & 0.012 \\
\hline Percent embryos out of total retrieved oocytes & $36 \pm 3.51$ & $50 \pm 4.73$ & 0.039 \\
\hline Count of doses & $25 \pm 1.31$ & $24 \pm 1.8$ & 0.79 [NS] \\
\hline
\end{tabular}

Note. NS: Not significant. Data presented as mean \pm standard error.

There was a significant direct correlation between the counts of the retrieved oocytes and serum FSH in the agonist protocol, but it was not statistically significant in the antagonist protocol. Moreover, a significant direct correlation was observed between the age and serum FSF in the antagonist protocol although it was not statistically significant in the agonist protocol (Table 6).

\section{Discussion}

In the normal menstrual cycle, GnRH stimulates the production of both $\mathrm{LH}$ and $\mathrm{FSH}$ from the pituitary gland. More precisely, both $\mathrm{LH}$ and FSH regulate follicle development and the subsequent production of the dominant follicle while ovulation is induced through the mid-cycle LH surge. In addition, the administration of GnRH stimulates LH and FSH above the critical levels required for the stimulation of multiple follicles which will be subsequently harvested for the IVF cycle. Further, the administration of GnRH agonist and antagonist prevents premature LH surge (by their effects on the pituitary gland that affects the naturally produced LH and FSH). The final step in the process is achieved using human chorionic 
Table 5. Multiple Logistic Regression Model With the Odds of Successful Chemical Pregnancy as the Dependent (Outcome) Variable by the Type of the Protocol Used After Adjusting for Age and the Selected Count Predictor

\begin{tabular}{lll}
\hline Predictors & Partial OR & $\boldsymbol{P}$ value \\
\hline Agonist protocol compared to antagonist & 1.57 & $0.23[\mathrm{NS}]$ \\
Count of M2 oocytes & 1.05 & $0.3[\mathrm{NS}]$ \\
Age & 1.03 & $0.36[\mathrm{NS}]$ \\
Count of transferred embryos & 1.88 & $<0.001$ \\
\hline
\end{tabular}

Note. Overall predictive accuracy $=72.5 \%$; $P$ value $($ model $)<0.001 ;$ NS: Non-significant; OR: odds ratio.

Table 6. The Relationship Between Serum FSH With Age, the Count of Doses, and the Count of Retrieved Oocytes

\begin{tabular}{lcccc}
\hline \multirow{2}{*}{ Variables } & \multicolumn{4}{c}{ Serum FSH } \\
\cline { 2 - 5 } & Agonist Protocol & Antagonist Protocol \\
\cline { 2 - 5 } & $\mathbf{r}$ & $\boldsymbol{P}$ Value & $\boldsymbol{r}$ & $\boldsymbol{P}$ Value \\
\hline Count of doses & 0.048 & $0.71[\mathrm{NS}]$ & 0.13 & $0.41[\mathrm{NS}]$ \\
Count of retrieved oocytes & 0.258 & 0.047 & -0.29 & $0.06[\mathrm{NS}]$ \\
Age (years) & 0.219 & $0.09[\mathrm{NS}]$ & 0.474 & 0.002 \\
\hline
\end{tabular}

Note. r: Correlation coefficient; FSH: follicle-stimulating hormone; NS: Non-significant.

gonadotropin bolus (GnRH agonist) and subsequent final oocyte maturation (18).

In the present study, the agonist arm showed a higher success of IVF outcomes with a 1.57 fold higher odds of achieving successful pregnancy compared to the antagonsit arm. However, it did not reach the statistical significance, which is in agreement with the findings of other clinical studies like Orvieto et al (16) favoring the use of the GnRH agonist protocol. Similarly, the findings are in line with those of the meta-analysis by Kolibianakis et al (19) in which GnRH agonist had higher odds of achieving live birth although it did not reach statistical significance (OR; 0.86, $95 \%$ CI: 0.72 to 1.02 , antagonist vs. agonist). In a more recent meta-analysis (20), the researchers examined 50 studies and found that the pregnancy rate in the $\mathrm{GnRH}$ antagonist arm had significantly lower odds compared to the GnRH agonist (relative risk: 0.89, 95\% CI: 0.82-0.96), which corroborates with our findings.

Several possible explanations are available for these findings. According to Kolibianakis et al (21), the LH surge is not sufficiently suppressed by the GnRH antagonist. In one study, the LH surge occurred in $8 \%$ of antagonist arms compared to $<1 \%$ in the agonist arm, and $80 \%$ of the surge in the antagonist arm occurred before the beginning of the antagonist therapy. Furthermore, low oocyte retrieval rates were obtained from the antagonist arm, which results in a lower pregnancy rate (22). This can be explained by the low suppression of endogenous FSH by the GnRH antagonist leading to asynchronous follicular development which is contrary to the appropriate suppression of FSH offered by the GnRH agonist resulting in better follicular development (23).

\section{Conclusions}

In general, the agonist protocol suggests better pregnancy outcomes compared to the antagonist protocol and the agonist protocol has an intrinsic benefit, which is not described by the higher number of transferred embryos.

\section{Authors' Contribution}

All author equally contributed to this work.

\section{Conflict of Interests}

Authors declare that they have no conflict of interests.

\section{Ethical Issues}

The Ethics Committee of Kamal AL-Samaria Hospital approved the study (ethical code: D201623). The purposes and procedures were explained to all participants and were given the right to participate or discontinue the study. Furthermore, written informed consent was taken, and patients were assured of data confidentially and the lack of using data for other research objects according to the Declaration of Helsinki (as revised in Edinburgh, 2000).

\section{Financial Support}

None.

\section{References}

1. Shokoohi M, Olad Saheb Madarek E, Khaki A, et al. Investigating the effects of onion juice on male fertility factors and pregnancy rate after testicular torsion/detorsion by intrauterine insemination method. Int J Women's Health Reprod Sci. 2018;6(4):499-505. doi:10.15296/ijwhr.2018.82

2. Porter RN, Smith W, Craft IL, Abdulwahid NA, Jacobs HS. Induction of ovulation for in-vitro fertilisation using buserelin and gonadotropins. Lancet. 1984;2(8414):12841285. doi:10.1016/s0140-6736(84)92840-x

3. Fleming R, Adam AH, Barlow DH, Black WP, MacNaughton MC, Coutts JR. A new systematic treatment for infertile women with abnormal hormone profiles. Br J Obstet Gynaecol. 1982;89(1):80-83. doi:10.1111/j.1471-0528.1982. tb04642.x

4. Majdi Seghinsara A, Shoorei H, Hassanzadeh Taheri $\mathrm{MM}$, et al. Panax ginseng extract improves follicular development after mouse preantral follicle 3D culture. Cell J. 2019;21(2):210-219. doi:10.22074/cellj.2019.5733

5. Trew G, Lavery S. Assisted reproduction. In: Edmonds DK, ed. Dewhurst's Textbook of Obstetrics \& Gynaecology. John Wiley \& Sons; 2012:580-494.

6. Shoorei H, Banimohammad M, Kebria MM, et al. Hesperidin improves the follicular development in $3 \mathrm{D}$ culture of isolated preantral ovarian follicles of mice. Exp Biol Med (Maywood). 2019;244(5):352-361. doi:10.1177/1535370219831615

7. Alizzi FJ, Showman HAK, Fawzi HA. Levonorgestrelreleasing intrauterine system in adenomyosis; predictors for response and clinical outcome. Asian J Pharm Clin Res. 2018;11(12):214-217. doi:10.22159/ajpcr.2018. v11i12.27109

8. Rizk B, Smitz J. Ovarian hyperstimulation syndrome after superovulation using GnRH agonists for IVF and related 
procedures. Hum Reprod. 1992;7(3):320-327. doi:10.1093/ oxfordjournals.humrep.a137642

9. Daya S. Gonadotropin releasing hormone agonist protocols for pituitary desensitization in in vitro fertilization and gamete intrafallopian transfer cycles. Cochrane Database Syst Rev. 2000(2):CD001299. doi:10.1002/14651858. cd001299

10. Olivennes F, Frydman R. Friendly IVF: the way of the future? Hum Reprod. 1998;13(5):1121-1124. doi:10.1093/ humrep/13.5.1121

11. Cheung LP, Lam PM, Lok IH, et al. GnRH antagonist versus long GnRH agonist protocol in poor responders undergoing IVF: a randomized controlled trial. Hum Reprod. 2005;20(3):616-621. doi:10.1093/humrep/deh668

12. Tarlatzis BC, Kolibianakis EM. GnRH agonists vs antagonists. Best Pract Res Clin Obstet Gynaecol. 2007;21(1):57-65. doi:10.1016/j.bpobgyn.2006.08.002

13. Rabinovici J, Rothman P, Monroe SE, Nerenberg C, Jaffe RB. Endocrine effects and pharmacokinetic characteristics of a potent new gonadotropin-releasing hormone antagonist (Ganirelix) with minimal histamine-releasing properties: studies in postmenopausal women. J Clin Endocrinol Metab. 1992;75(5):1220-1225. doi:10.1210/ jcem.75.5.1385467

14. Qublan HS, Amarin Z, Tahat YA, Smadi AZ, Kilani M. Ovarian cyst formation following GnRH agonist administration in IVF cycles: incidence and impact. Hum Reprod. 2006;21(3):640-644. doi:10.1093/humrep/dei371

15. Kumar P, Sharma A. Gonadotropin-releasing hormone analogs: understanding advantages and limitations. J Hum Reprod Sci. 2014;7(3):170-174. doi:10.4103/09741208.142476

16. Orvieto R, Rabinson J, Meltzer S, Homburg R, Anteby E, Zohav E. GnRH agonist versus GnRH antagonist in ovarian stimulation: is the emperor naked? Clin Exp Obstet Gynecol. 2006;33(4):197-199.

17. Rubino P, Viganò P, Luddi A, Piomboni P. The ICSI procedure from past to future: a systematic review of the more controversial aspects. Hum Reprod Update. 2016;22(2):194-227. doi:10.1093/humupd/dmv050

18. Alper MM, Fauser BC. Ovarian stimulation protocols for IVF: is more better than less? Reprod Biomed Online. 2017;34(4):345-353. doi:10.1016/j.rbmo.2017.01.010

19. Kolibianakis EM, Collins J, Tarlatzis BC, Devroey P, Diedrich K, Griesinger G. Among patients treated for IVF with gonadotrophins and GnRH analogues, is the probability of live birth dependent on the type of analogue used? a systematic review and meta-analysis. Hum Reprod Update. 2006;12(6):651-671. doi:10.1093/humupd/dml038

20. Lambalk CB, Banga FR, Huirne JA, et al. GnRH antagonist versus long agonist protocols in IVF: a systematic review and meta-analysis accounting for patient type. Hum Reprod Update. 2017;23(5):560-579. doi:10.1093/humupd/dmx017

21. Kolibianakis EM, Venetis CA, Kalogeropoulou L, Papanikolaou E, Tarlatzis BC. Fixed versus flexible gonadotropin-releasing hormone antagonist administration in in vitro fertilization: a randomized controlled trial. Fertil Steril. 2011;95(2):558-562. doi:10.1016/j. fertnstert.2010.05.052

22. Nelson SM, Yates RW, Fleming R. Serum anti-Müllerian hormone and FSH: prediction of live birth and extremes of response in stimulated cycles--implications for individualization of therapy. Hum Reprod. 2007;22(9):24142421. doi:10.1093/humrep/dem204

23. Huirne JA, Homburg R, Lambalk CB. Are GnRH antagonists comparable to agonists for use in IVF? Hum Reprod. 2007;22(11):2805-2813. doi:10.1093/humrep/ dem 270

(c) 2020 The Author(s); This is an open-access article distributed under the terms of the Creative Commons Attribution License (http:// creativecommons.org/licenses/by/4.0), which permits unrestricted use, distribution, and reproduction in any medium, provided the original work is properly cited. 